\title{
Isotopenaustausch-Untersuchungen an Silicat-Ionenaustauschern
}

\author{
V. Die Kinetik der Selbstdiffusion von Alkalimetall-Ionen in synthetischem Zeolith vom Typ A
}

E. Hoinkis und H. W. Levi

Hahn-Meitner-Institut für Kernforschung Berlin, Sektor Kernchemie

(Z. Naturforschg. 22 a, 226-232 [1967] ; eingegangen am 27. September 1966)

\begin{abstract}
Gemessen wurden Selbstdiffusionsprozesse in den Na-, K-, Rb- und Cs-Formen von synthetischem Zeolith A. Der Na- und K-Isotopenaustausch verläuft extrem schnell. Zur Auswertung des Rb- und Cs-Austauschs wurde eine für Würfel mit normalverteilten Kantenlängen geeignete Methode angewandt. Beide Austauschprozesse sind keine einheitlichen, idealen Diffusionsprozesse. Sie ließen sich jedoch widerspruchsfrei auswerten unter der Annahme, daß zwei verschieden schnelle Diffusionsprozesse unabhängig voneinander nebeneinander ablaufen. Der Anteil des langsamen Teilprozesses am Gesamtprozeß nimmt mit steigender Temperatur ab. Die Aktivierungsenergien der beiden Teilprozesse sind fast gleich, die $D_{0}$-Werte unterscheiden sich um 2 Größenordnungen. Die Existenz zweier Diffusionsprozesse wird der Tatsache zugeschrieben, daß die austauschfähigen Ionen im Gitter des Zeolith A in zwei strukturell ungleichwertigen Positionen untergebracht sind.
\end{abstract}

Untersuchungen dieser Art an reinem kristallinen Linde Molekular Sieb (LMS) A liegen bisher nicht vor. Der Grund hierfür dürfte die hohe Austauschgeschwindigkeit sein, die durch die Feinkörnigkeit der verfügbaren Präparate und die relativ hohen Selbstdiffusionskoeffizienten bedingt ist. Lediglich Ames ${ }^{1}$ hat Selbstdiffusionskoeffizienten für Alkalimetallionen bestimmt, dafür aber Preßkörper mit hohem Bindergehalt verwendet. Die Ergebnisse sind daher sicher nicht auf reine Kristalle übertragbar. Außerdem haben Freeman und Stamires ${ }^{2}$ Selbstdiffusionskoeffizienten von Alkalimetallionen aus Leitfähigkeitsmessungen an wasserfreien Zeolithen vom Typ X und A berechnet. Diese Untersuchungen hatten ergeben, daß im Typ $X$ zwei verschiedene Aktivierungsenergien der Selbstdiffusion existieren, die die Autoren auf die ungleichwertige Unterbringung der austauschfähigen Kationen im Gitter zurückführen.

Es schien nun reizvoll zu untersuchen, ob sich ein solches Phänomen auch durch direkte Diffusionsmessungen mittels heterogenem Isotopenaustausch nachweisen läßt. Für diese Untersuchungen wurde zunächst der synthetische Zeolith Typ A gewählt, bei dem ebenso wie beim Typ $\mathrm{X}$ austauschfähige Kationen auf verschiedenen Gitterpositionen untergebracht sind.

Dyer und Fawcett ${ }^{3}$ haben die Selbstdiffision

1 L. L. Ames JR., Am. Mineralogist 50, 465 [1965].

2 D. C. Freeman Jr. u. D. N. Stamires, J. Chem. Phys. 35, 799 [1961].

3 A. Dyer u. J. M. Fawcett, J. Inorg. Nucl. Chem. 28, 615 [1966]. von Erdalkaliionen in diesem Zeolith studiert. Sie haben jedoch nur jeweils die ersten 30\% der Austauschprozesse gemessen und dabei keinen Hinweis auf einen uneinheitlichen Verlauf gefunden. Ein wesentliches Charakteristikum der in dieser Arbeit beschriebenen Untersuchungen ist die Auswertung der vollständigen Austauschprozesse.

Die Struktur des synthetischen Zeoliths Typ A ist von Reed und Breck ${ }^{4}$, Barrer und Meier ${ }^{5}$ sowie Broussard und Shoemaker ${ }^{6}$ diskutiert worden. Alle drei Arbeiten kommen in den wesentlichen Punkten zum gleichen Ergebnis. Strukturelement sind die aus $\mathrm{SiO}_{4}$ - bzw. $\mathrm{AlO}_{4}$-Tetraedern aufgebauten Kubooktaeder des Sodaliths, von denen je einer die acht Ecken der kubischen Elementarzelle bildet. Dadurch entsteht ein Hohlraum von $11,4 \AA$ Durchmesser, der durch Sauerstoff-8-Ringe von 4,2 $\AA$ Durchmesser zugänglich ist.

Die Kubooktaeder sind über ihre quadratischen Flächen durch vier Sauerstoffatome verbunden und kehren ihre hexagonalen Flächen (Sauerstoff-6Ringe) dem Innern des großen Hohlraumes zu.

Die 12 austauschfähigen Na-Ionen sind zum Teil in einer 8-zähligen Punktlage untergebracht, die sich zentrisch auf den hexagonalen Flächen der Kubooktaeder, also aus den Sauerstoff-6-Ringen herausgerückt, befindet. Die übrigen sind nach Broussard und SHozmaker nicht lokalisierbar, d. h. statistisch

4 T. B. Reed u. D. W. Breck, J. Amer. Chem. Soc. 78, 5972 [1956].

5 R. M. Barrer u. W. M. Meier, Trans. Faraday Soc. 54, 1074 [1958].

${ }^{6}$ L. Broussard u. D. P. Shoemaker, J. Amer. Chem. Soc. 82. 1041 [1960]. 
in der Elementarzelle verteilt. Nach REED und BRECK sitzen sie in den Sauerstoff-8-Ringen, die die $\mathrm{Zu}$ gänge zu den großen Käfigen bilden und sind statistisch auf diese verteilt.

Nach Barrer und MeIer ist ein zusätzliches $\mathrm{NaAlO}_{2}$ im Innern der kleinen Kubooktaeder okkludiert. Da diese Hohlräume durch Sauerstoff-6Ringe von nur 2,0 $\AA$ Durchmesser zugänglich sind, kann dieses Na-Ion durch große Ionen wie $\mathrm{Tl}^{+}$nicht ersetzt werden. Entsprechend wurde für $\mathrm{Tl}^{+}$nur 12/13 der Na-Kapazität gefunden.

\section{Voruntersuchungen}

\subsection{Bestimmung der Korngröße}

Die mikroskopische Betrachtung der LMS A-Kriställchen zeigte, daß die Körner ziemlich exakt würfelförmig sind, jedoch keine einheitliche Größe haben. Die Körner wurden daher in Größenklassen eingeteilt und ausgezählt. Im Wahrscheinlichkeitsnetz ergab die Summenhäufigkeit aufgetragen gegen die obere Klassengrenze mit guter Annäherung eine Gerade, so daß man die Korngrößenverteilung als Normalverteilung (GAuss-Verteilung) behandeln kann. Der Mittelwert der Würfelkante ergibt sich zu $a_{\mathrm{m}}=4,3 \mu$, die Standardabweichung ist $\sigma_{0}=1,7$, d. h. $s=a_{\mathrm{m}} / \sqrt{2} \sigma_{0}=1,8$.

Diese Bestimmung wurde an der käuflichen NaForm des Zeolithes (LMS A 4) durchgeführt, es wurde aber sichergestellt, daß durch Beladung mit anderen Ionen keine Änderung der Korngröße eintrat.

\subsection{Bestimmung der Austauschkapazitäten}

Zur Bestimmung der Beladungskapazitäten und gleichzeitig zur Herstellung der bei den Isotopenaustauschversuchen verwendeten radioaktiven Austauscherproben wurde LMS A 4 mit den betreffenden radioaktiv markierten Ionen beladen. Hierzu wurden eine größere Menge des Austauschers sowie eine in ein Zentrifugenglas eingewogene kleine Probe in genau gleicher Weise bei $95^{\circ}$ mit einer 1-n. radioaktiv markierten Lösung bekannter spezifischer Aktivität des betreffenden Chlorids behandelt. Die Lösungen wurden so oft erneuert, bis nach ca. 1-tägigem Kontakt mit dem Austauscher an der kleinen Probe keine Aktivitätszunahme mehr festzustellen war. Aus den in der kleinen Probe gemessenen Radioaktivitätswerten ergaben sich die in Tab. 1 zusammengestellten Beladungskapazitäten für
$\mathrm{K}, \mathrm{Rb}, \mathrm{Cs}$ und die Isotopenaustauschkapazität für das ursprünglich im Austauscher enthaltene Na.

\begin{tabular}{|c|c|c|c|c|}
\hline Ion $\mathrm{X}^{+}$ & $\mathrm{Na}$ & $\mathrm{Rb}$ & $\mathrm{Cs}$ & $\mathrm{K}$ \\
\hline $\begin{array}{l}\text { Kapazität } \\
\text { für X }\end{array}$ & 5,5 & 5,10 & 3,15 & 5,5 \\
$\begin{array}{c}\text { Kap. für X+ } \\
\text { Kap. für Na }\end{array}$ & - & $12,1: 13$ & $7,4: 13$ & $13: 13$ \\
$\begin{array}{l}\text { Ionendurch- } \\
\text { messer }(\AA)\end{array}$ & 1.94 & 2,94 & 3,34 & 2,66 \\
\hline
\end{tabular}

Tab. 1. Kapazität von LMS A 4.

Es war zu erwarten, daß Rb-Ionen, die den gleichen Radius wie $\mathrm{Tl}(\mathrm{I})$-Ionen haben, wie diese nur $12 / 13$ des $\mathrm{Na}$ zu ersetzen vermögen. Überraschend ist es dagegen, daß $\mathrm{K}$ alle $\mathrm{Na}$-Ionen ersetzen kann. $\mathrm{K}$-Ionen sind zwar kleiner als Rb- und Tl-Ionen, haben aber mit 2,94 $\AA$ immer noch einen größeren Durchmesser als die $2 \AA$-Fenster der kleinen Käfige. Die K-Ionen müssen daher erheblich deformiert werden, um in diese Käfige eindringen zu können. Erwartungsgemäß äußert sich dies in der Kinetik der K-Beladung. Bei $20^{\circ} \mathrm{C}$ wurden $12 / 13$ der NaIonen in wenigen Minuten durch $\mathrm{K}$ ersetzt, während der Rest nach Tagen noch nicht vollständig ausgetauscht war.

Die gegenüber der $\mathrm{Tl}$ - und Rb-Kapazität noch geringere Cs-Kapazität dürfte zwar ohne Zweifel mit dem größeren Radius des Cs-Ions zusammenhängen, sie ist jedoch nicht durch Unterbringungsschwierigkeiten zu erklären. Rein geometrisch hätten auch 12 Cs-Ionen in einer Elementarzelle reichlich Platz. Der Grund könnte in einer weitergehenden Entwässerung beim Ersatz des $\mathrm{Na}$ durch $\mathrm{Cs}$ gesucht werden, die durch die geringe Hydratisierungstendenz des großen Cs-Ions bedingt ist. Damit wird die Coulombsche Abstoßung zwischen den zusammengedrängten Cs-Ionen stärker wirksam und eine Anordnung von $12 \mathrm{Cs}$ in der Elementarzelle energetisch ungünstig.

\subsection{Ermittlung des geschwindigkeitsbestimmenden Reaktionsschrittes}

Wegen der hohen Isotopenaustausch-Geschwindigkeit der Alkaliformen von Zeolith sollte experimentell sichergestellt werden, daß Festkörperdiffusion der geschwindigkeitsbestimmende Schritt des Austauschs ist. Für diese Versuche wurden die Stabmethode und die Druckfiltrationsmethode verwendet (vgl. IV. Mitteilung ${ }^{7}$ ).

7 E. Hoinkis, H. W. Levi, W. Lutze, W. Miekeley u. T. TAMBERG, Z. Naturforschg. 22 a, 220 [1967]. 
Abb. 1 zeigt Austauschkurven mit langsam und mit schnell $(4000 \mathrm{U} / \mathrm{min})$ gedrehtem Stab in 0,1und 0,001-n. CsCl-Lösung sowie Druckfiltrationswerte mit 0,1 - und 1-n. CsCl-Lösung. Man sieht, daß ab 0,1-n. weder die Drehgeschwindigkeit des Stabes (Dicke des hydrodynamischen Grenzfilmes) noch die Konzentration der Lösung einen Einfluß auf die Austauschgeschwindigkeit haben. In 0,001-n. Lösung wirkt sich die Drehgeschwindigkeit dagegen deutlich aus. Bei einer Konzentration von 0,1-n. und darüber ist also sicher Korndiffusion, bei 0,001-n. und darunter sicher Filmdiffusion geschwindigkeitsbestimmend. Die folgenden Isotopenaustauschversuche wurden mit 1-n. CsCl-Lösung gemacht.

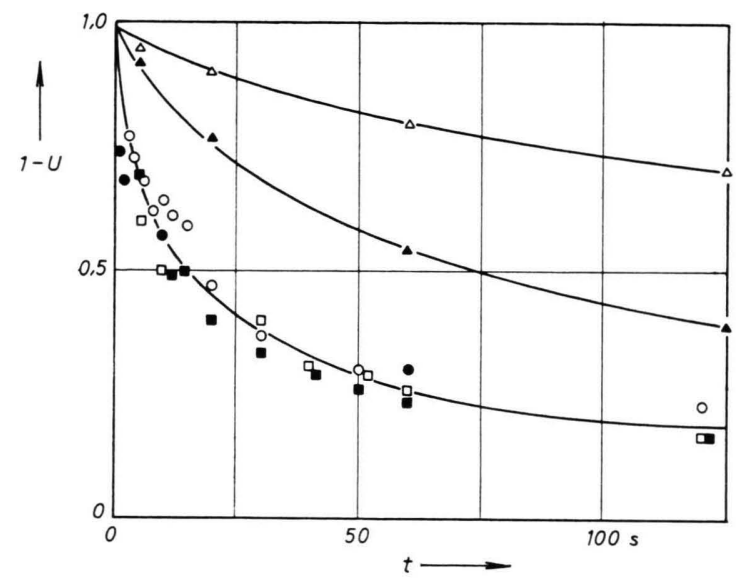

Abb. 1. Bestimmung des geschwindigkeitsbestimmenden Schrittes beim Cs-Isotopenaustausch in Zeolith A: $(1-U)=f(t)$. Stabmethode:

$\triangle 40 \mathrm{U} / \mathrm{min}, \boldsymbol{\Delta} 4000 \mathrm{U} / \mathrm{min}, 0,001-n$. CsCl-Lösung,

$\bigcirc 0 \mathrm{U} / \mathrm{min}$,

Druckfiltration: $4000 \mathrm{U} / \mathrm{min}, 0,1-n$. CsCl-Lösung. 0,1-n., $\mathbf{1 , 0 - n}$.

\section{Messung des Isotopenaustausches}

Für die Messung des Isotopenaustausches wurden Anordnungen verwendet, die in der IV. Mitteilung ${ }^{7}$ beschrieben sind. Für alle Versuche mit kurzen Austauschzeiten wurde die Druckfiltrationsmethode benutzt. Die übrigen Versuche wurden konventionell durchgeführt, und zwar durch Schütteln von Festkörper und Lösung in einem Zentrifugenglas. Die Lösung enthielt jeweils mindestens die 100-fache Ionenmenge wie der Festkörper, so daß die Überschußbedingung gut erfüllt war. Die Aktivität der Lösung bei Versuchsbeginn war Null. Gemessen wurde in allen Fällen die Aktivität des Festkörpers nach Abtrennen von der Lösung. Hierzu wurde ein
Szintillations-Meßplatz mit Bohrlochkristall und Einkanaldiskriminator benutzt.

Bestimmt wurde der Umsatzbruchteil $U$ in Abhängigkeit von der Zeit, und zwar für den Cs- und RbAustausch bei je vier Temperaturen zwischen 0 und $30{ }^{\circ} \mathrm{C}$. Der Temperaturbereich war nach oben durch die zunehmende Geschwindigkeit des Austausches begrenzt.

Der Umsatzgrad $U$ ergibt sich aus der gemessenen Restaktivität im Festkörper zu

$$
U=\left(\overline{A_{0}}-\overline{A_{t}}\right) /\left(\bar{A}_{0}-\bar{A}_{\infty}\right),
$$

$\overline{A_{0}}, \overline{A_{\infty}}, \overline{A_{t}}$ sind die Radioaktivitäten des Festkörpers zur Zeit $t=0, t=\infty$ bzw. zur Meßzeit $t$.

$\mathrm{Rb}$ wird wesentlich schneller ausgetauscht als Cs. $\mathrm{Na}$ und K werden so schnell ausgetauscht, daß diese Prozesse sich mit den gegenwärtig verfügbaren $\mathrm{Me}$ methoden nicht genau genug messen lassen, um sie auswerten zu können. Beim Na konnte aber festgestellt werden, daß bei $0^{\circ}$ nach ca. 30 sec $92 \%$ umgesetzt sind, während der Austausch der restlichen Ionen sich über viele Minuten hinzog. Das entspricht der erwähnten Unterbringung von 1/13 der Na-Ionen in den kleinen Sodalith-Käfigen.

\section{Auswertung des Cs-Isotopenaustauschs}

Als Grundlage für die Auswertung dient die in der IV. Mitteilung ${ }^{7}$ angegebene Lösung der Diffusionsgleichung für eine Menge von würfelförmigen Körnern mit normalverteilten Kantenlängen. Mit Hilfe des dort wiedergegebenen Diagramms $\log (1-U)=f\left(\tau^{2}\right)$ wurden die Meßwerte $U(t)$ in ein $\tau^{2}(t)$-Diagramm übertragen (Abb. 2). In diesem Diagramm sollte sich für einen einheitlichen idealen

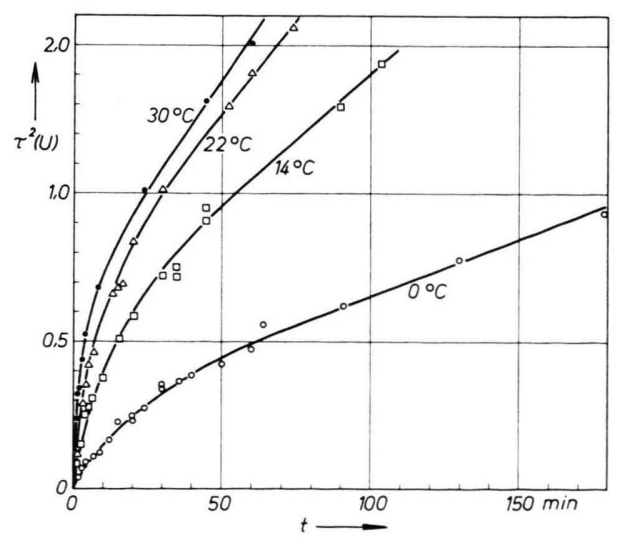

Abb. 2. Cs-Isotopenaustausch in Zeolith A: $\tau^{2}(U)=f(t)$. 
Diffusionsprozeß eine Gerade mit der Steigung $\left(16 / a_{\mathrm{m}}{ }^{2} \pi\right) D$ ergeben. Wie die Abbildung zeigt, ist dies jedoch nicht der Fall, sondern man erhält stark gekrümmte Kurven. Diese Kurven haben aber am Ende einen geraden Teil.

Es handelt sich also offenbar nicht um einen idealen einheitlichen Diffusionsprozeß. Es wurde nun der Versuch gemacht, den Prozeß so zu behandeln, als liefen zwei verschieden schnelle einheitliche, ideale Diffusionsprozesse unabhängig voneinander nebeneinander ab. Die Veranlassung, einen Versuch gerade mit dieser Behandlungsweise zu machen, war in erster Linie die Tatsache, daß die austauschfähigen Kationen in zwei strukturell ungleichwertigen Gitterpositionen untergebracht sind. Es besteht eine deutliche Analogie zu Austauschprozessen, bei denen ein Teil der an sich austauschfähigen Kationen so langsam ausgetauscht werden, daß vor Erreichen des vollständigen Umsatzes die Austauschgeschwindigkeit praktisch Null wird. In diesen Fällen ist das Nebeneinander zweier sich kinetisch verschieden verhaltender Ionenanteile evident.

Für einen derart zusammengesetzten Austauschprozeß wurde angesetzt

$$
U=(1-y) U_{\mathrm{s}}+y U_{\mathrm{l}} .
$$

Darin ist $(1-y)$ der Anteil des Gesamtprozesses, der auf den schnellen Teilprozeß entfällt, und $U_{\mathrm{s}}$ und $U_{1}$ sind die Umsatzbruchteile der isoliert betrachteten Teilprozesse. Zu bestimmen sind die Parameter $y, D_{\mathrm{s}}$ und $D_{\mathrm{l}}$.

\subsection{Bestimmung von $y$}

Den Anteil des schnellen Teilprozesses kann man aus $\ln (1-U)=f(t)$ bestimmen. Diese Funktion hat bei einheitlichem Diffusionsprozeß eine gerade Asymptote, die für $U>0,75$ den Verlauf der Kurve gut wiedergibt. Sie wird bei Vorliegen einer Normalverteilung im $\log (1-U)=f(t)$-Diagramm durch die Gleichung

$$
\log (1-U)=\log k_{1}-1,107 \frac{16}{\pi a_{\mathrm{m}}^{2}} D t
$$

beschrieben. Die Verlängerung dieser Asymptote schneidet also die Ordinate bei $\log k_{1}$. Den Wert für $k_{1}$ bei einer Normalverteilung mit $s=1,8$ entnimmt man der Abb. 1 in der IV. Mitteilung ${ }^{7} \mathrm{zu} k_{1}=0,435$.

Bei einem zusammengesetzten Diffusionsprozeß kann die Funktion $\ln (1-U)=f(t)$ erst dann in die gerade Asymptote übergehen, wenn $U_{\mathrm{s}} \approx 1$, die schnelle Teilreaktion also praktisch keinen zeitlich veränderlichen Beitrag mehr zum Gesamtprozeß leistet. Es ist dann $(1-U)=y\left(1-U_{1}\right)$. Man erhält also:

$$
\log (1-U)=\log \left(y k_{1}\right)-1,107 \frac{16}{\pi a_{\mathrm{m}}^{2}} D t .
$$

In diesem Falle schneidet die Verlängerung der Asymptote die Ordinate nicht in $\log k_{1}$, sondern in $\log \left(y k_{1}\right)$. Man kann also auf diese Weise den Anteil $1-y$ der schnellen Reaktion bestimmen. Das ist in Abb. 3 ausgeführt. Man findet, daß der Anteil der schnellen Reaktion mit steigender Temperatur zunimmt.

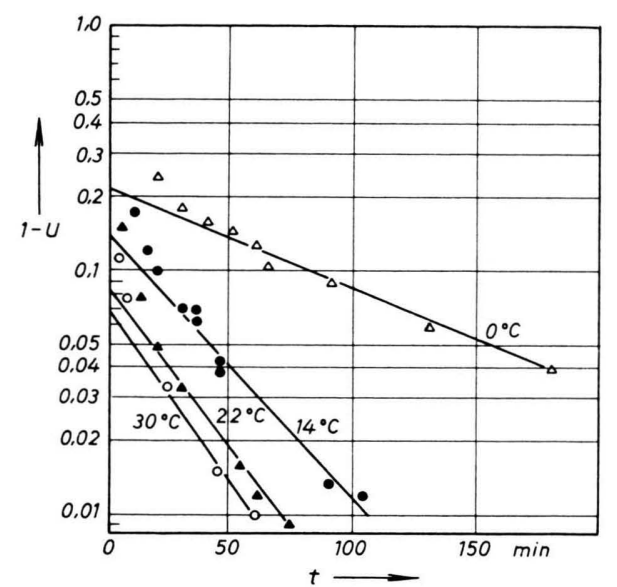

Abb. 3. Cs-Isotopenaustausch in Zeolith A: $\log (1-U)=f(t)$.

\subsection{Bestimmung von $D_{1}$}

Den Diffusionskoeffizienten des langsamen Teilprozesses kann man direkt aus der Steigung der Asymptote von $\log (1-U)=f(t)$ bestimmen. Nach Gl. (3) ist diese dem Diffusionskoeffizienten $D_{1}$ proportional.

Generell ist es aber vorzuziehen, $D_{1}$ aus der Steigung des geraden Astes am Ende von $\tau^{2}(U)=f(t)$ zu berechnen, der der Asymptote von $\log (1-U)=f(t)$ hinsichtlich der Steigung äquivalent ist. $\tau^{2}(U)=f(t)$ kann nämlich am Ende erst dann geradlinig werden, wenn die schnelle Reaktion praktisch keinen zeitlich veränderlichen Beitrag mehr leistet, da $\tau^{2}\left(U_{1}+U_{\mathrm{s}}\right)$ nicht proportional $\tau^{2}\left(U_{1}\right)+\tau^{2}\left(U_{\mathrm{s}}\right)$ ist. Der Vorteil liegt darin, daß für die lineare Asymptote die Bedingungen $U_{\mathrm{s}} \approx 1$ und $U_{1} \approx 0,75$ erfüllt sein müssen, für den linearen Bereich am Ende von $\tau^{2}(U)=f(t)$ dagegen nur die Bedingung $U_{\mathrm{s}} \approx 1$. Daher kann man in günstigen Fällen einen größeren Umsatzbereich für die Bestimmung von $D_{1}$ verwenden. 
Es ist also:

$$
D_{1}=\frac{\tau^{2}\left[U\left(t_{2}\right)\right]-\tau^{2}\left[U\left(t_{1}\right)\right]}{t_{2}-t_{1}} \cdot \frac{a_{\mathrm{m}}^{2}}{16}
$$

unter der Voraussetzung, daß $\mathrm{d} \tau^{2} / \mathrm{d} t=$ const für $t>t_{1}$.

\subsection{Bestimmung von $D_{\mathrm{s}}$}

$\mathrm{Um} D_{\mathrm{s}}$ zu bestimmen, muß man $\tau^{2}\left(U_{\mathrm{s}}\right)=f_{\mathrm{s}}(t)$ auftragen, d. h. man muß

$$
U_{\mathrm{s}}(t)=\frac{U(t)-y U_{1}(t)}{1-y}
$$

berechnen. $\tau^{2}\left(U_{1}\right)=f_{1}(t)$ und damit $U_{1}(t)$ erhält man, indem man das verlängerte gerade Endstück von $\tau^{2}(U)=f(t)$, aus dessen Steigung $D_{1}$ erhalten wurde, so verschiebt, daß es durch den Koordinatenursprung geht. $\tau^{2}\left(U_{\mathrm{s}}\right)=f_{\mathrm{s}}(t)$ muß eine Gerade ergeben.

Es ist dann:

$$
D_{\mathrm{s}}=\frac{\tau^{2}\left[U_{\mathrm{S}}\left(t_{2}{ }^{\prime}\right)\right]-\tau^{2}\left[U_{\mathrm{s}}\left(t_{1}{ }^{\prime}\right)\right]}{t_{2}^{\prime}-t_{1}^{\prime}} \cdot \frac{a_{\mathrm{m}}{ }^{2}}{16} .
$$

In Abb. 4 ist das ganze Verfahren an einem Beispiel dargestellt. Für die anderen Temperaturen ist das Bild entsprechend.

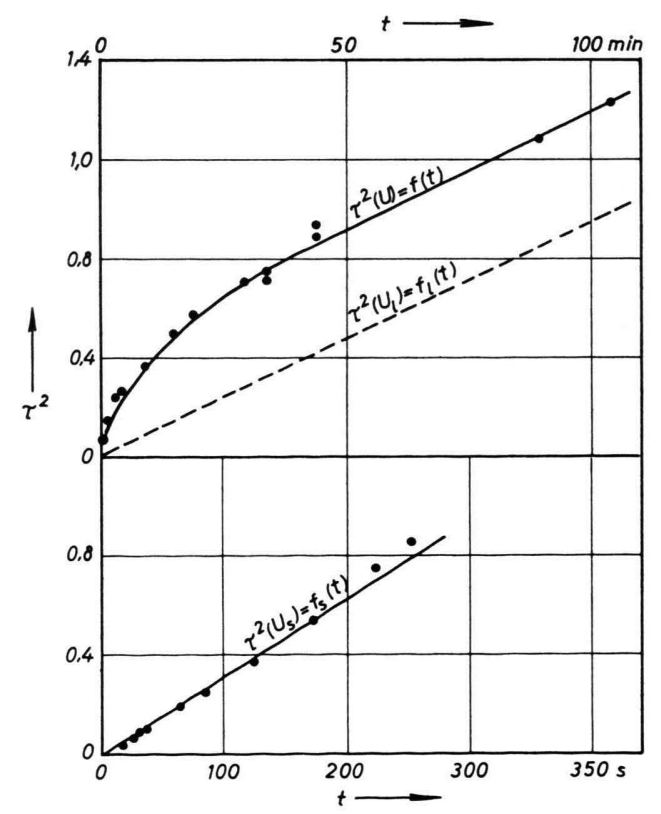

Abb. 4. Cs-Isotopenaustausch in Zeolith A bei $14^{\circ}$; Zerlegung von $\tau^{2}(U)=f(t)$ in $\tau^{2}\left(U_{\mathrm{s}}\right)=f_{\mathrm{s}}(t)$ und $\tau^{2}\left(U_{1}\right)=f_{1}(t)$.

Die Abbildung demonstriert auch, daß diese Zerlegung des experimentell beobachteten Austauschprozesses aus einer nicht im ganzen Umsatzbereich linea- ren Funktion $\tau^{2}(U)=f(t)$ je eine lineare Funktion $\tau^{2}\left(U_{\mathrm{s}}\right)=f_{\mathrm{s}}(t)$ und $\tau^{2}\left(U_{1}\right)=f_{1}(t)$ macht.

\subsection{Numerische Auswertung}

Die hier durchgeführte Bestimmung von $y, D_{\mathrm{s}}$ und $D_{1}$ muß korrekterweise als Abschätzung bezeichnet werden, da wegen des schnellen Verlaufs des Austauschprozesses und der geringen Unterschiede von $D_{\mathrm{s}}$ und $D_{1}$ nur wenige Meßpunkte für die Bestimmung von $y$ und $D_{1}$ zur Verfügung stehen. Es wurde daher der Versuch gemacht, den ganzen Verlauf der Austauschkurven auf Grund der gefundenen Parameter mit Hilfe eines elektronischen Rechners zu berechnen. Hierzu wurden in die Gleichung

$$
U(t)=(1-y) U_{\mathrm{s}}(t)+y U_{1}(t)
$$

als Umsatzfunktionen die exakte Lösung der Diffusionsgleichung für Würfel mit normalverteilter Kantenlänge mit $s=1,8$ (vgl. IV. Mitteilung ${ }^{7}$ ) eingesetzt. Mit geringfügiger Variation dieser Parameter erhält man befriedigende Übereinstimmung zwischen den berechneten und den gemessenen Austauschkurven.

In Abb. 5 sind die Meßpunkte sowie die berechneten Kurven eingezeichnet. In Tab. 2 sind $y, D_{\mathrm{s}}$ und $D_{1}$ zusammengestellt.

\subsection{Berechnung von Aktivierungsenergien}

Die Logarithmen der beiden Diffusionskoeffizienten wurden gegen $1 / T$ aufgetragen (Abb. 6). In allen Fällen resultieren mit guter Annäherung Geraden, aus denen sich die ebenfalls in Tab. 2 enthaltenen Aktivierungsenergien ergeben.

\begin{tabular}{|c|c|c|c|}
\hline $\begin{array}{c}\text { Temp. } \\
{ }^{\circ} \mathrm{C}\end{array}$ & $y$ & $\begin{array}{c}D_{1} \cdot 10^{12} \\
\mathrm{~cm}^{2} \mathrm{sec}^{-1}\end{array}$ & $\begin{array}{c}D_{\mathrm{s}} \cdot 10^{10} \\
\mathrm{~cm}^{2} \mathrm{sec}^{-1}\end{array}$ \\
\hline 0 & 0,50 & 2,2 & 0,8 \\
14 & 0,32 & 4,1 & 1,4 \\
22 & 0,20 & 5,4 & 1,9 \\
30 & 0,16 & 6,8 & 2,6 \\
\hline $\begin{array}{c}E_{A} \\
\mathrm{kcal} / \mathrm{Mol}\end{array}$ & - & 6,5 & 6,2 \\
\hline $\begin{array}{c}D_{0} \\
\mathrm{~cm}^{2} \mathrm{sec}^{-1}\end{array}$ & - & $3 \cdot 10^{-7}$ & $6 \cdot 10^{-5}$ \\
\hline
\end{tabular}

Tab. 2. Cs-Selbstdiffusion in Zeolith A.

\section{Rb-Isotopenaustausch}

$\tau^{2}(U)=f(t)$ für den Rb-Isotopenaustausch gibt ein ganz ähnliches Bild wie für Cs (Abb. 7). Die Auswertung ist wesentlich unsicherer, da der Prozeß so 


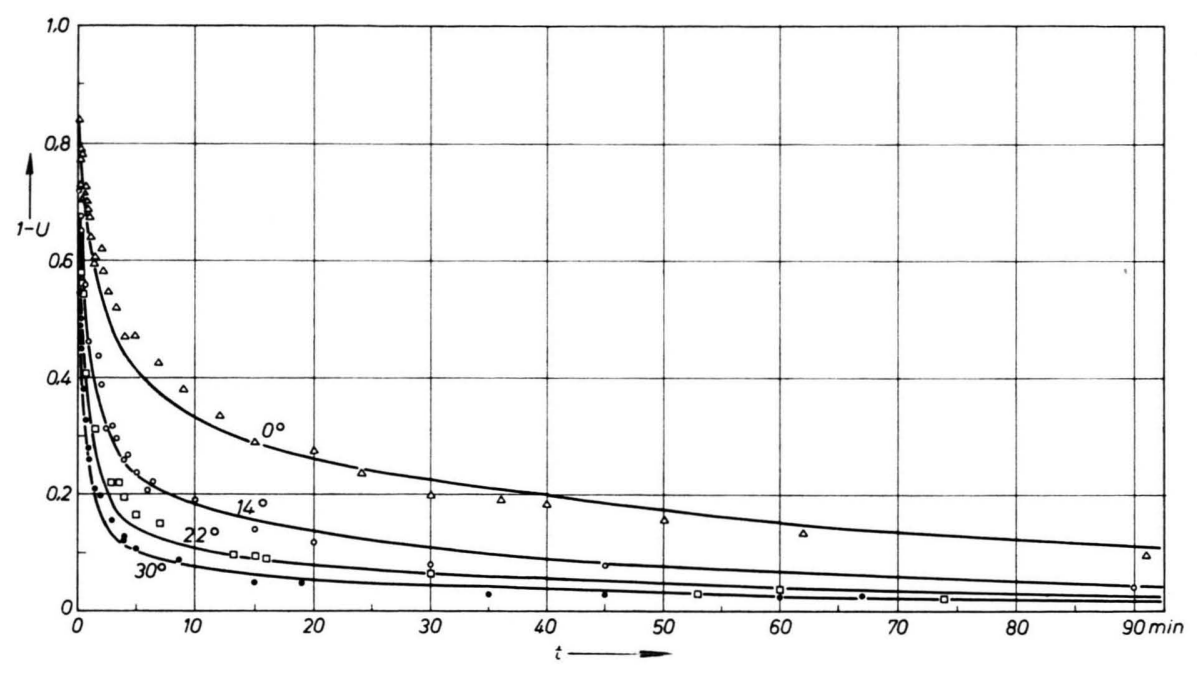

Abb. 5. Cs-Isotopenaustausch in Zeolith A, Vergleich des berechneten Ablaufs (Kurven) mit dem gemessenen (Punkte).

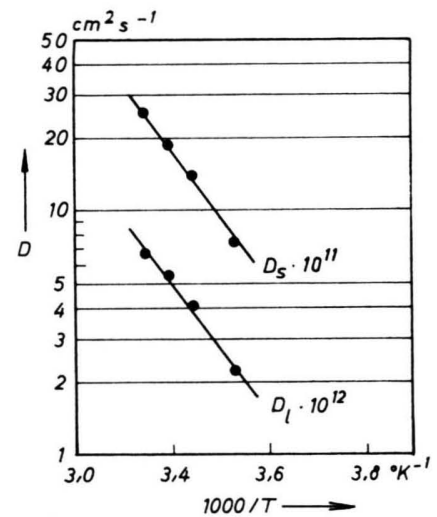

Abb. 6. Temperaturabhängigkeit der Cs-Selbstdiffusionskoeffizienten in Zeolith A.

schnell ist, daß er an der Grenze des überhaupt noch Auswertbaren liegt. Die Anteile des langsamen und des schnellen Teilprozesses am Gesamtprozeß sind ungefähr die gleichen wie bei Cs. Die Diffusionskoeffizienten des langsamen Teilprozesses sind ungefähr um den Faktor 10, die des schnellen um den Faktor 5 größer als die entsprechenden Werte für Cs. Die Aktivierungsenergien sind etwas kleiner als bei Cs.

\section{Diskussion}

Die folgende Diskussion hat vorläufigen Charakter, da sie auf der allerdings sehr eingehenden Untersuchung eines einzigen Isotopenaustauschprozesses in synthetischem Zeolith A basiert.

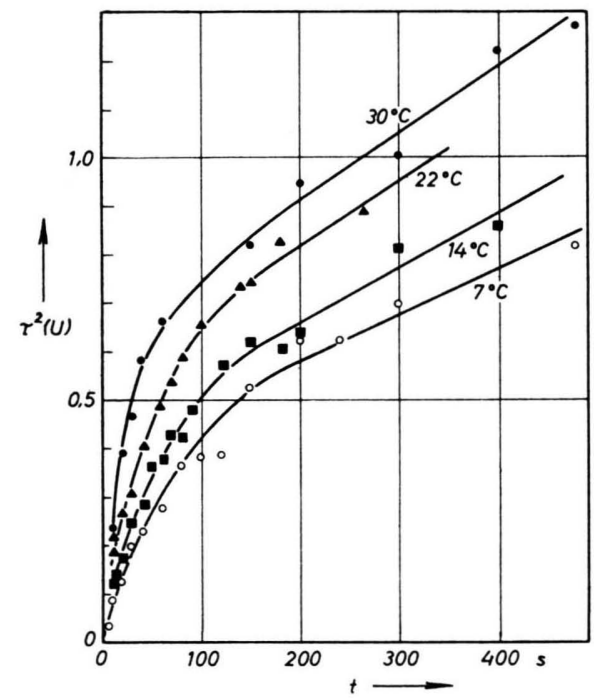

Abb. 7. Rb-Isotopenaustausch in Zeolith A: $\tau^{2}(U)=f(t)$.

Der experimentell beobachtete Cs-Isotopenaustauschprozeß ließ sich vollständig in zwei Teilprozesse zerlegen, die beide mit hinreichender Genauigkeit die Kriterien eines idealen Diffusionsprozesses erfüllen. Sie liefern beide lineare Funktionen $\tau^{2}(U)=f(t)$. Die Aktivierungsenergien der beiden Teilprozesse sind fast gleich, die $D_{0}$-Werte unterscheiden sich um zwei Größenordnungen. Der Anteil der Ionen, der nach dem langsamen Prozeß diffundiert, nimmt mit steigender Temperatur ab.

Ausgangspunkt für die formale Zerlegung in zwei einzelne Diffusionsprozesse war die Tatsache, daß die austauschfähigen Kationen im Zeolith A auf zwei 
strukturell ungleichwertige Gitterpositionen verteilt sind. Man müßte demzufolge annehmen, daß die verschieden untergebrachten Ionen unabhängig voneinander diffundieren. Gestützt wird diese Vermutung dadurch, daß im Na-Zeolith A ein extrem langsamer Prozeß beobachtet (vgl. ${ }^{2}$ ), wenn auch nicht ausgewertet wurde, der ganz offensichtlich der in dieser Ionenform des Zeolith A vorkommenden dritten Unterbringungsart zuzuordnen ist.

Die Temperaturabhängigkeit der Verteilung auf die beiden Diffusionsprozesse läßt in diesem Bild auf ein thermisches Gleichgewicht zwischen den beiden Gitterpositionen schließen. Da bei höherer Temperatur der schnell austauschende Anteil begünstigt ist, ist dessen Energieniveau im Gitter offenbar das höhere. Sicherlich sind die auf den Sauerstoff-6-Ringen der Kubooktaeder angeordneten Ionen die fester gebundenen. Da von diesen Positionen nur acht zur Verfügung stehen, dürfte der langsam diffundierende Anteil 2/3 nicht überschreiten. Das ist in dem hier untersuchten Temperaturbereich auch nicht der Fall $\left(50 \%\right.$ bei $\left.0{ }^{\circ} \mathrm{C}\right)$.

Um zu verstehen, daß die Aktivierungsenergie des langsamen Diffusionsprozesses nicht höher ist als die des schnellen, könnte man die Annahme machen, $\mathrm{da} ß$ die langsam diffundierenden Ionen bei ihrer Verschiebung weitgehend im Coulombschen Anziehungsbereich der negativen Festladungen (Sauerstoffatome) bleiben. Das ist insofern plausibel, als sie nach der Strukturbestimmung sozusagen einseitig an der hexagonalen Kubooktaederfläche kleben. Sie können also an dieser entlang über die Brücken-Sauerstoffatome zur nächsten gleiten. Es wäre daher denkbar, daß die hierfür aufzuwendende Aktivierungsenergie so klein ist, daß die Hauptbarriere für beide Prozesse die gleiche, nämlich das Passieren der $4 \AA$-Fenster (Sauerstoff-8-Ringe) zwischen den großen Käfigen ist. In den $D_{0}$-Werten, die ja die Ursache für die große Differenz zwischen den Diffusionskonstanten sind, würde sich dann die durch die Verschieden- artigkeit der Gleichgewichtspositionen bedingte Unterschiedlichkeit der Diffusionsmechanismen manifestieren. Verschieden ist sicher die Distanz des Elementarsprungs. Außerdem dürften aber auch die Schwingungsfrequenz in der Gleichgewichtsposition und die Diffusionsentropie andere Werte haben.

Barrer und Mitarbeiter ${ }^{8}$ fanden am Chabasit, dessen effektive Porenweite die gleiche ist wie bei Zeolith A, eine Cs-Aktivierungsenergie von $7,5 \mathrm{kcal}$ $/$ mol und einen $D_{0}$-Wert in der Größenordnung von $10^{-5} \mathrm{~cm}^{2} \mathrm{sec}^{-1}$. Der von Barrer gemessene Diffusionsprozeß im Chabasit entspricht also offenbar weitgehend dem schnellen Teilprozeß im Zeolith A. Allerdings fand BARRER keine so ausgeprägten Geschwindigkeitsunterschiede zwischen den einzelnen Alkaliionen.

Die aus der Zerlegbarkeit des Isotopenaustauschprozesses in zwei unabhängige Diffusionsprozesse sich ergebende Folgerung, daß die in verschiedenen Gitterpositionen untergebrachten Kationen unabhängig voneinander im Kristall diffundieren, ist überraschend. Man hätte eher erwartet, daß sich innerhalb des Kristalls ein Isotopenaustauschgleichgewicht zwischen den beiden Gitterpositionen einstellt. Die Möglichkeit einer ganz anderen Beschreibung der Selbstdiffusion im Zeolith A soll daher nicht ausgeschlossen werden. Untersuchungen an den Erdalkaliformen sowohl dieses Zeoliths als auch des Zeolith X, die im Gange sind, sollen helfen, das Problem endgültig zu klären.

Die Versuche wurden zum Teil von Frau R. Peuckert und Herrn W. Hensel ausgeführt. - Wir danken Herrn Prof. K. E. Zimen für die Förderung der Arbeit. Dem Sektor Mathematik des Hahn-Meitner-Instituts danken wir für die numerische Berechnung von Austauschkurven. - Die Arbeit wurde aus ERP-Forschungsmitteln des Senators für Wirtschaft unterstützt.

8 R. M. Barrer, R. F. Bartholomew u. L. V. C. Rees, J. Phys. Chem. Solids 24, 51 [1963]. 